\title{
Groups of $S$-units in Hyperelliptic Fields
}

\author{
V. V. Benyash-Krivets ${ }^{a}$ and Academician V. P. Platonov ${ }^{b}$ \\ Received August 1, 2007
}

DOI: $10.1134 / \mathrm{S} 106456240706021 \mathrm{X}$

In this paper, we calculate groups of $S$-units in hyperelliptic fields.

Let $k=F_{q}(x)$ be the field of rational functions of one variable over a finite field $F_{q}$ of characteristic $p>2$, and let

$$
d(x)=a_{0} x^{2 n+1}+a_{1} x^{2 n}+\ldots+a_{2 n+1}
$$

be a square-free polynomial with $a_{0} \neq 0$. Consider $K=$ $k(\sqrt{d})$. For an irreducible polynomial $v \in F_{q}[x]$, by ||$_{V}$ we denote the corresponding valuation on $k$, by $O_{v}=$ $\left\{\left.z \in k|| z\right|_{V} \geq 0\right\}$ the ring of the valuation ||$_{V}$, and by $p_{V}=$ $\left\{\left.z \in k|| z\right|_{V}>0\right\}$ the ideal of the valuation $|\cdot|_{V}$. The residue field $k_{\mathrm{V}}=O_{v} / p_{\mathrm{v}}$ coincides with $F_{p}[x] /(\mathrm{v})$ and is a finite extension of $F_{p}$. Let $\bar{x}$ be the image of $x$ in residue field $k_{V}$ If $d(\bar{x})=\beta^{2}$ for some $0 \neq \beta \in k_{V}$ (this means that $(\beta, \bar{x})$ is a $k_{\mathrm{v}}$-point of the hyperelliptic curve $\left.y^{2}=d(x)\right)$, then the valuation $|\cdot|_{V}$ admits two nonequivalent extensions to the field $K$. We denote these valuations by $|\cdot|_{v^{\prime}}$ and ||$_{V}$. Otherwise, the valuation ||$_{V}$ has a unique extension to the field $K$, which we denote by the same symbol $|\cdot|_{v}$, The non-Archimedean valuation $|\cdot|_{\infty}$ admits a unique extension to $K$; we denote it by $|\cdot|_{\infty}$.

Let $S$ be an arbitrary finite set of nonequivalent valuations of the field $K$ containing $|\cdot|_{\infty}$, and let $S_{1}=\left\{|\cdot|_{\infty}\right.$, $\left.|\cdot|_{v_{1}}, \ldots,|\cdot|_{v_{t}}\right\}$ be the set of restrictions of valuations from $S$ to the field $k$. We use $O_{S}$ to denote the ring of $S$-integer elements in $K$, i.e., of all $z \in K$ such that $|z|_{V} \geq 0$ for all valuations $|\cdot|_{V}$ of $K$ not belonging to $S$. The set $U_{S}$ of invertible elements of the ring $O_{S}$ is called the group of $S$-units of the field $K$. By virtue of the generalized Dirichlet theorem about units (see [1, Chapter IV, The-

\footnotetext{
${ }^{a}$ Belarussian State University, pr. Nezavisimosti 4, Minsk, 220030 Belarus e-mail:benyash@bsu.by

${ }^{b}$ Research Institute for System Studies, Russian Academy of Sciences, Nakhimovskii pr. 36, korp. 1,

Moscow, 117218 Russia

e-mail: platonov@niisi.ras.ru
}

orem 9]), the group $U_{S}$ is the direct product of the group $F_{q}^{*}$ and the free Abelian group $G$ of rank $|S|-1$. Independent generators of the group $G$ are called fundamental $S$-units.

In the classical case of the quadratic extension $L=$ $Q(\sqrt{d})$ of the field $Q$, a fundamental unit of the field $L$ can be found by expanding $\sqrt{d}$ into a continued fraction [2]. However, for functional fields, the method of continued fractions does not always yield a fundamental unit. The purpose of this paper is to construct an algorithm for calculating fundamental $S$-units of a hyperelliptic field $K$.

The first proposition is of technical character.

Proposition 1. Let $y=f+g \sqrt{d}$, where $f, g \in F_{q}[x]$; $f \neq 0 ; g \neq 0 ;$ and $(f, g)=1$, and let $v \in F_{q}[x]$ be an irreducible polynomial.

Then, the following assertions hold.

(i) If ||$_{V}$ admits two extensions ||$_{V^{\prime}}$ and ||$_{V^{\prime \prime}}$ to $K$, then either $|y|_{V^{\prime}}=0$ or $|y|_{V^{\prime \prime}}=0$.

(ii) If $\vee \chi d$ and ||$_{V}$ admits a unique extension $|\cdot|_{V}$ to $K$, then $|y|_{V}=0$.

(iii) If $v \mid d$ and $v \backslash f$, then $|\cdot|_{V}$ admits a unique extension to $K$, and $|y|_{V}=0$.

(iv) If $v \mid d$ and $v \mid f$, then ||$_{V}$ admits a unique extension to $K$, and $|y|_{V}=\frac{1}{2}$.

The following proposition characterizes $S$-integer elements in $K$.

Proposition 2. Any element $y \in O_{S}$ has the form

$$
y=\frac{f+g \sqrt{d}}{V_{1}^{m_{1}} V_{2}^{m_{2}} \ldots V_{t}^{m_{t}}},
$$

where $f, g \in F_{q}[x]$ and $m_{i} \geq 0$. If some $m_{i}$ is positive, then $v_{i} \backslash$ f and $v_{i} \backslash g$. 
To prove Proposition 2, it suffices to note that if the denominator of $y$ is divisible by an irreducible polynomial $V \neq V_{i}$, where $i=1,2, \ldots, t$, then, by Proposition 1 , we have $|y|_{V^{\prime}}<0$ for some extension $|\cdot|_{V^{\prime}}$ of the valuation $|\cdot|_{V}$.

Note that not every element of the form $y=$ $\frac{f+g \sqrt{d}}{V_{1}^{m_{1}} V_{2}^{m_{2}} \ldots V_{t}^{m_{t}}}$ is an $S$-integer. For what follows, it is important to know what values a valuation mapping can take at $S$-units.

Proposition 3. If $\varepsilon \in U_{s}$ and $\varepsilon \notin F_{q}^{*}$, then

$$
N_{K / k}(\varepsilon)=a v_{1}^{m_{1}} V_{2}^{m_{2}} \ldots v_{t}^{m_{t}},
$$

where $a \in F_{q}^{*}, m_{i} \in \mathbf{Z}$, and $m_{1}, m_{2}, \ldots, m_{t}$ are not all zero.

As in the case of $S$-integer elements, an element $\varepsilon \in$ $K$ satisfying the condition $N_{K / k}(\varepsilon)=a v_{1}^{m_{1}} V_{2}^{m_{2}} \ldots v_{t}^{m_{t}}$ is not necessarily an $S$-unit.

$$
\text { If } \varepsilon=\frac{f+g \sqrt{d}}{V_{1}^{m_{1}} V_{2}^{m_{2}} \ldots v_{t}^{m_{t}}} \in U_{S} \backslash F_{q}^{*} \text {, then it follows from }
$$

Proposition 3 that

$$
f^{2}-g^{2} d=a v_{1}^{k_{1}} v_{2}^{k_{2}} \ldots v_{t}^{k_{t}},
$$

where $k_{1}, k_{2}, \ldots, k_{t}$ are nonnegative integers. The following proposition shows that if the valuation equation (1) with fixed $k_{1}, k_{2}, \ldots, k_{t}$ has a solution in polynomials $f$, $g \in F_{q}[x]$, then we can easily construct an $S$-unit.

Proposition 4. Suppose that $z=f+g \sqrt{d} \in K$, where $f, g \in F_{q}[x]$, and

$$
N_{K / k}(z)=f^{2}-g^{2} d=a v_{1}^{m_{1}} V_{2}^{m_{2}} \ldots v_{t}^{m_{t}},
$$

where $a \in F_{q}^{*}, m_{i} \in \mathbf{Z}$, and $m_{1}, m_{2}, \ldots, m_{t}$ are not all zero. Let $S_{2}=\left\{|\cdot|_{v_{1}},|\cdot|_{v_{2}}, \ldots,|\cdot|_{v_{r}}\right\}$ be the set of those valuations $|\cdot|_{V_{i}}$ from $S_{1}$ for which the following conditions hold: (i) $|\cdot|_{V_{i}}$ admits two extensions $|\cdot|_{V_{i}^{\prime}}$ and $|\cdot|_{V_{i}^{\prime \prime}}$ to $K$; (ii) $|\cdot|_{V_{i}^{\prime \prime}} \notin S$; (iii) $|z|_{v_{i}^{\prime \prime}}>0$.

$$
\text { Then, } \frac{z}{V_{1}^{m_{1}} V_{2}^{m_{2}} \cdots V_{r}^{m_{r}}} \in U_{S} \text {. }
$$

Now, consider the natural question of how a system of independent fundamental $S$-units expands when the set $S$ is augmented with a new valuation ||$_{v}$. It is answered by the following theorem.
Theorem 1. Suppose that $f=|S|-1, \varepsilon_{1}, \varepsilon_{2}, \ldots, \varepsilon_{f}$ are independent fundamental $S$-units of the field $K$ and $v \in$ $F_{q}[x]$ is an irreducible polynomial.

Then, the following assertions hold.

(i) Suppose that the valuation ||$_{V}$ admits two extensions $|\cdot|_{V^{\prime}}$ and $|\cdot|_{V^{\prime \prime}}$ to $K$ and, moreover, $|\cdot|_{V^{\prime}} \in S$ and $|\cdot|_{v^{\prime \prime}} \notin S$. Let $S^{\prime}=S \cup\left\{|\cdot|_{v^{\prime \prime}}\right\}$. Then, $\varepsilon_{1}, \varepsilon_{2}, \ldots, \varepsilon_{f}$, vform a system of independent fundamental $S^{\prime}$-units.

(ii) Suppose that the valuation ||$_{V}$ admits two extensions ||$_{v^{\prime}}$ and ||$_{v^{\prime \prime}}$ to $K$ and, moreover, ||$_{v^{\prime}} \notin S$ and ||$_{v^{\prime \prime}} \notin S$. Let $S^{\prime}=S \cup\left\{|\cdot|_{V^{\prime \prime}}\right\}$, and let $\varepsilon_{f+1}$ be an $S^{\prime}$-unit for which

$$
N_{K / k}\left(\varepsilon_{f+1}\right)=a v_{1}^{m_{1}} V_{2}^{m_{2}} \ldots V_{f}^{m_{f}} V_{f+1}^{m_{f+1}},
$$

where $m_{f+1}$ is the minimum possible positive integer exponent. Then, $\varepsilon_{1}, \varepsilon_{2}, \ldots, \varepsilon_{f}, \varepsilon_{f+1}$ form a system of independent fundamental $S$ '-units.

(iii) Let $v \mid d$. Then, the valuation ||$_{V}$ admits a unique extension to K. Suppose that $|\cdot|_{V} \notin S$ and $S^{\prime}=S \cup\left\{|\cdot|_{V}\right\}$. If $\frac{d}{V} \notin F_{q}$, then $\varepsilon_{1}, \varepsilon_{2}, \ldots, \varepsilon_{f}, V$ form a system of independent fundamental $S^{\prime}$-units. If $\frac{d}{V} \in F_{q}$, then $\varepsilon_{1}, \varepsilon_{2}, \ldots$, $\varepsilon_{f}, \sqrt{d}$ form a system of independent fundamental $S^{\prime}$-units.

It follows from Theorem 1 that the key case in finding a system of independent fundamental $S$-units is as follows. Let $v_{1}, v_{2}, \ldots, v_{t} \in F_{q}[x]$ be irreducible polynomials such that each valuation $|\cdot|_{V_{i}}$ admits two extensions $|\cdot|_{v_{i}^{\prime}}$ and $|\cdot|_{V_{i}^{\prime}}$ to $K$. We set $S=\left\{|\cdot|_{\infty},|\cdot|_{V_{1}^{\prime}}, \ldots\right.$, $|\cdot|_{V_{t}^{\prime}}$ \}, i.e., include precisely one of the two extensions of $|\cdot|_{v_{i}}$ to $K$ in $S$.

First, consider the minimal case, in which $S=\left\{|\cdot|_{\infty}\right.$, $\left.|\cdot|_{v^{\prime}}\right\}$. If $\varepsilon \in U_{S}$, then, by Proposition 3 , we have $N_{K / k}(\varepsilon)=a v^{m}$. Therefore, to calculate a fundamental $S$-unit, we must find the minimum positive integer $m$ for which the valuation equation

$$
f^{2}-g^{2} d=a v^{m},
$$

where $a \in F_{q}^{*}$, has a solution in polynomials $f, g \in$ $F_{q}[x]$. By virtue of Proposition 4, either $f+g \sqrt{d}$ or $f-$ $g \sqrt{d}$ is a fundamental $S$-unit.

The most complete result is obtained when $v=x-a$ is a first-degree polynomial. Let $\bar{k}_{V}$ be the completion of $k$ with respect to the valuation $|\cdot|_{V}$. The field $\bar{k}_{V}$ can be identified with the field $F_{q}((v))$ of formal power series. Since ||$_{V}$ admits two extension to $K$, it follows 
that $\sqrt{d} \in F_{q}((v))$. The following theorem provides an algorithm for finding fundamental $S$-units.

Theorem 2. Suppose that $\sqrt{d}=\sum_{i=0}^{\infty} d_{i} v^{i} \in F_{q}((v))$, $n=\frac{\operatorname{deg} d-1}{2}, r \geq n$ is an integer, and

$$
D_{r}=\left(\begin{array}{cccc}
d_{n+1} & d_{n+2} & \ldots & d_{r+1} \\
d_{n+2} & d_{n+3} & \ldots & d_{r+2} \\
& & \ddots & \\
d_{n+r} & d_{n+r+1} & \ldots & d_{2 r}
\end{array}\right)
$$

and

$$
H_{r}=\left(\begin{array}{cccc}
d_{n+2} & d_{n+3} & \ldots & d_{r+2} \\
d_{n+3} & d_{n+4} & \ldots & d_{r+3} \\
& & \ddots & \\
d_{n+r+1} & d_{n+r+2} & \ldots & d_{2 r+1}
\end{array}\right)
$$

are matrices. Then, the valuation equation (2) with odd $m=2 r+1$ has a solution in polynomials $f, g \in F_{q}[x]$ if and only if $r \geq n$ and $\operatorname{rank} D_{r}<r-n+1$, and the valuation equation (2) with even $m=2 r$ has such a solution if and only if $r \geq n+1$ and $\operatorname{rank} H_{r-1}<r-n$.

Proof. Suppose that $m=2 r+1$ (the case of even $m$ is considered similarly). Let $f, g \in F_{q}[x]$ be a solution to (2). Then, $\operatorname{deg} f \leq r$ and $\operatorname{deg} g=r-n$. Expanding $f$ and $g$ in powers of $V$, we obtain

$$
\begin{gathered}
f=f_{0}+f_{1} V+\ldots+f_{r} V^{r}, \\
g=g_{0}+g_{1} v+\ldots+g_{r-n} V^{r-n},
\end{gathered}
$$

where $f_{i}, g_{j} \in F_{q}$. Thus,

$$
g \sqrt{d}=\sum_{i=0}^{\infty} P_{i} V^{i}
$$

where $P_{i}=\sum_{j=0}^{i} g_{j} d_{i-j}$ (we assume that $g_{j}=0$ for $j>r-n$ ).

The valuation equation (2) is equivalent to $f+g \sqrt{d}=$ $\sum_{i=m}^{\infty} P_{i} v^{i}$, which gives the following systems of linear equations for the coefficients $f_{i}$ and $g_{j}$ :

$$
\begin{gathered}
f_{i}=-P_{i}, \quad i=0,1, \ldots, r-1, \\
P_{j}=0, \quad j=r, r+1, \ldots, m-1 .
\end{gathered}
$$

Setting $G=\left(g_{r-n}, g_{r-n-1}, \ldots, g_{0}\right)^{t}$, we can rewrite (4) in the matrix form

$$
D_{r} G=0 .
$$

Since the homogeneous system (5) of linear equations with matrix $D_{r}$ has a nonzero solution $G$, it follows that $\operatorname{rank} D_{r}<r-n+1$.

Conversely, if rank $D_{r}<r-n+1$, then (5) has a nonzero solution $G$. Formulas (3) give the coefficients $f_{i}$; thus, we obtain polynomials $f$ and $g$ such that $v^{m} \mid f^{2}-$ $g^{2} d$. By construction, $\operatorname{deg}\left(f^{2}-g^{2} d\right) \leq \operatorname{deg} v^{m}$ and, obviously, $f^{2}-g^{2} d \neq 0$; hence, $f^{2}-g^{2} d=a v^{m}$, where $a \in F_{q}^{*}$.

Thus, to find a fundamental $S$-unit of the field $K$, we expand $\sqrt{d}$ in a power series in the field $k_{V}((v))$. Then, successively calculating the ranks of the matrices $D_{r}$ and $H_{r}$, we find a minimum positive integer $r$ for which either $\operatorname{rank} D_{r}<r-n+1$ or rank $H_{r-1}<r-n$. After that, solving the homogeneous system of linear equations (5), we obtain a nonzero polynomial $g$, and formulas (3) give a polynomial $f$. The required fundamental $S$-unit has the form $f+g \sqrt{d}$.

If $K$ is the function field of an elliptic curve, i.e., $\operatorname{deg} d=3$, then the matrices $D_{r}$ and $H_{r}$ are square, and Theorem 2 acquires the following form.

Corollary. Suppose that $\operatorname{deg} d=3$. Then, the valuation equation (2) with odd $m=2 r+1$ has a solution in polynomials $f, g \in F_{q}[x]$ if and only if $\operatorname{det} D_{r}=0$, and the valuation equation (2) with even $m=2 r$ has such a solution if and only if $\operatorname{det} H_{r-1}=0$.

In the case where $\operatorname{deg} v \geq 2$, the problem of finding a fundamental $S$-unit also reduces to solving a homogeneous system of linear equations. However, the matrix of this system is hard to write out explicitly. In this case, to solve the valuation equation (2), we write

$$
f=f_{0}+f_{1} x+\ldots+f_{r} x^{r}
$$

and

$$
g=g_{0}+g_{1} x+\ldots+g_{e} x^{e},
$$

where $r=\left[\frac{m \operatorname{deg} v}{2}\right]$ and $e=\left[\frac{m \operatorname{deg} v-\operatorname{deg} d}{2}\right]([z]$ denotes the integer part of $z$ ). Since $\sqrt{d} \in \bar{k}_{\mathrm{V}}$, it follows that the elements $f$ and $g \sqrt{d}$ can be represented as the formal power series

$$
f=f_{0}^{\prime}+f_{1}^{\prime} v+\ldots+f_{r^{\prime}}^{\prime} V^{r^{\prime}} \quad \text { and } \quad g \sqrt{d}=\sum_{i=0}^{\infty} L_{i} v^{i}
$$

DOKLADY MATHEMATICS Vol. 76 No. 32007 
where the coefficients $f_{i}^{\prime}$ and $L_{i}$ are polynomials from $F_{q}[x]$ of degree $<\operatorname{deg} v$, moreover, the coefficients of $f_{i}^{\prime}$ are linear forms in $f_{0}, f_{1}, \ldots, f_{r}$ and the coefficients of $L_{i}$ are linear forms in $g_{0}, g_{1}, \ldots, g_{e}$. Let us require that $f+$

$$
\begin{aligned}
& g \sqrt{d}=\sum_{i=m}^{\infty} L_{i} v^{i}, \text { i.e., } \\
& f_{0}^{\prime}=-L_{0}, \quad f_{1}^{\prime}=-L_{1}, \ldots, f_{r^{\prime}}^{\prime}=-L_{r^{\prime}}, \\
& L_{r^{\prime}+1}=L_{r^{\prime}+2}=\ldots=L_{m-1}=0 .
\end{aligned}
$$

Relations (7) give a homogeneous system of linear equations with respect to $g_{0}, g_{1}, \ldots, g_{e}$ with some matrix $A_{V}$ :

$$
A_{\mathrm{v}}\left(g_{0}, g_{1}, \ldots, g_{e}\right)^{t}=0 \text {. }
$$

System (8) has a nonzero solution if and only if $\operatorname{rank} A_{V} \leq e$. If this condition holds, then we find the polynomial $g$ from (8) and the polynomial $f$ from (6). By construction, $v^{m} \mid f^{2}-g^{2} d, \operatorname{deg}\left(f^{2}-g^{2} d\right) \leq \operatorname{deg} v^{m}$, and, obviously, $f^{2}-g^{2} d \neq 0$. Therefore, $f^{2}-g^{2} d=a v^{m}$, where $a \in F_{q}^{*}$.

Now, let $S=\left\{|\cdot|_{\infty},|\cdot|_{v_{1}^{\prime}}, \ldots,|\cdot|_{v_{t}^{\prime}}\right\}$. According to assertion 2 of Theorem 1, a system of independent fundamental $S$-units can be constructed by induction. Let $S_{i}=\left\{|\cdot|_{\infty},|\cdot|_{v_{1}^{\prime}}, \ldots,|\cdot|_{v_{i}^{\prime}}\right\}$. Using Theorem 2, we find a fundamental $S_{1}$-unit. Suppose that $\varepsilon_{1}, \varepsilon_{2}, \ldots, \varepsilon_{i}$ are independent fundamental $S_{i}$-units and

$$
\begin{gathered}
N_{K / k}\left(\varepsilon_{j}\right)=a_{j} v_{1}^{m_{j 1}} v_{2}^{m_{j 2}} \ldots v_{j}^{m_{j j}}, \quad a_{j} \in F_{q}^{*}, \\
j=1,2, \ldots, i .
\end{gathered}
$$

Adding an $S_{i+1}$-unit $\varepsilon_{i+1}$ with minimum possible positive integer exponent $m_{i+1, i+1}$, we obtain independent fundamental $S_{i+1}$-units.

Let $\varepsilon_{1}, \varepsilon_{2}, \ldots, \varepsilon_{t}$ be independent fundamental $S$-units thus constructed. Consider the valuation matrix

$$
H\left(\varepsilon_{1}, \varepsilon_{2}, \ldots, \varepsilon_{t}\right)=\left(\begin{array}{cccc}
m_{11} & 0 & \ldots & 0 \\
m_{21} & m_{22} & \ldots & 0 \\
& \ddots & \\
m_{t 1} & m_{t 2} & \ldots & m_{t t}
\end{array}\right) .
$$

If $\varepsilon_{1}^{\prime}, \varepsilon_{2}^{\prime}, \ldots, \varepsilon_{t}^{\prime}$ for another system of independent fundamental $S$-units, then

$$
\varepsilon_{i}^{\prime}=\varepsilon_{1}^{b_{i 1}} \varepsilon_{2}^{b_{i 2}} \ldots \varepsilon_{t}^{b_{i t}}, \quad i=1,2, \ldots, t
$$

and $B=\left(b_{i j}\right) \in G L_{t}(\mathbf{Z})$. It is easy to see that

$$
H\left(\varepsilon_{1}^{\prime}, \varepsilon_{2}^{\prime}, \ldots, \varepsilon_{t}^{\prime}\right)=B H\left(\varepsilon_{1}, \varepsilon_{2}, \ldots, \varepsilon_{t}\right) .
$$

Therefore, multiplying $H\left(\varepsilon_{1}, \varepsilon_{2}, \ldots, \varepsilon_{t}\right)$ by a suitable matrix $B \in G L_{t}(\mathbf{Z})$ if necessary, we can assume that $0 \leq$ $m_{i r}<m_{r r}$ for $i=r+1, r+2, \ldots, t$. Let $T_{i}=\left\{|\cdot|_{\infty},|\cdot|_{\mathrm{v}_{i}^{\prime}}\right\}$, and let $\delta_{i}$ be the corresponding fundamental $T_{i}$-unit. Then, $N_{K / k}\left(\delta_{i}\right)=b_{i} v_{i}^{m_{i}}$, where $b_{i} \in F_{q}^{*}$. Since $\delta_{i}$ is an $S_{i}$-unit, it follows that $\delta_{i}=c_{i} \varepsilon_{1}^{f_{1}} \varepsilon_{2}^{f_{2}} \ldots \varepsilon_{i}^{f_{i}}$, where $c_{i} \in$ $F_{q}^{*}$. Comparing the values of the left- and right-hand sides, we obtain $f_{i} m_{i i}=m_{i}$, i.e., $m_{i i} \mid m_{i}$.

Suppose that the units $\varepsilon_{1}, \varepsilon_{2}, \ldots, \varepsilon_{i-1}$ are already found. To find $\varepsilon_{i}$, we must determine the minimum positive integer divisor $m_{i i}$ of $m_{i}$ and integers $0 \leq m_{i j}<m_{j j}$, where $j=1,2, \ldots, i-1$, for which the valuation equation

$$
f^{2}-g^{2} d=a v_{1}^{m_{i 1}} v_{1}^{m_{i 2}} \ldots v_{i}^{m_{i i}},
$$

where $a \in F_{q}^{*}$, has a solution in polynomials $f, g \in$ $F_{q}[x]$. As in the case of one valuation, solving (9) reduces to solving a homogeneous system of linear equations. It follows from (9) that

$$
\operatorname{deg} f=\left[\frac{1}{2} \sum_{j=1}^{i} m_{i j} \operatorname{deg} v_{j}\right]=l
$$

and

$$
\operatorname{deg} g=\left[\frac{1}{2}\left(\sum_{j=1}^{i} m_{i j} \operatorname{deg} v_{j}-\operatorname{deg} d\right)\right]=e .
$$

Suppose that $f=f_{0}+f_{1} x+\ldots+f_{l} x^{l}$ and $g=g_{0}+g_{1} x+\ldots+$ $g_{e} x^{e}$. Choose one of the valuations $|\cdot|_{V_{j}}$, where $1 \leq j \leq i$. Let us represent $f+g \sqrt{d}$ as a formal power series in $v_{j}$ :

$$
f+g \sqrt{d}=\sum_{s=0}^{\infty} L_{s} v_{j}^{s},
$$

where $L_{s} \in F_{q}[x]$ and $\operatorname{deg} L_{s}<\operatorname{deg} v_{j}$; the coefficients of the polynomial $L_{s}$ are linear forms in $f_{0}, f_{1}, \ldots, f_{l}, g_{0}$, $g_{1}, \ldots, g_{e}$. We require that

$$
L_{0}=L_{1}=\ldots=L_{m_{i j}-1}=0 .
$$

Then, (10) yields a homogeneous system of linear equations with respect to $f_{0}, f_{1}, \ldots, f_{l}, g_{0}, g_{1}, \ldots, g_{e}$ with matrix $A_{v_{j}}$ such that

$$
A_{V_{j}}\left(f_{0}, f_{1}, \ldots, f_{l}, g_{0}, g_{1}, \ldots, g_{e}\right)^{t}=0 \text {. }
$$

After performing this construction for all valuations $|\cdot|_{v_{j}}$ with $j=1,2, \ldots, i$, we see that $f_{0}, f_{1}, \ldots, f_{l}$, $g_{0}, g_{1}, \ldots, g_{e}$ are a solution to the homogeneous system of linear equations

$$
A\left(f_{0}, f_{1}, \ldots, f_{l}, g_{0}, g_{1}, \ldots, g_{e}\right)^{t}=0,
$$


where $A$ is a block matrix of the form $A=\left(A_{v_{1}}, A_{v_{2}}, \ldots\right.$, $\left.A_{\mathrm{V}_{i}}\right)^{t}$.

Conversely, if $f_{0}, f_{1}, \ldots, f_{l}, g_{0}, g_{1}, \ldots, g_{e}$ are a solution to (12) and not all of the $g_{j}$ vanish, then the nonzero polynomial $f^{2}-g^{2} d$ is divisible by the product $v_{1}^{m_{i 1}} v_{2}^{m_{i 2}} \ldots v_{i}^{m_{i i}}$. By construction, we have $\operatorname{deg} f^{2}-$ $g^{2} d \leq \operatorname{deg} v_{1}^{m_{i 1}} v_{2}^{m_{i 2}} \ldots v_{i}^{m_{i i}}$; therefore, $f^{2}-g^{2} d=$ $a v_{1}^{m_{i 1}} V_{2}^{m_{i 2}} \ldots v_{i}^{m_{i i}}$, where $a \in F_{q}^{*}$. Thus, we have proved the following theorem.
Theorem 3. The valuation equation (9) has a solution in nonzero polynomials $f, g \in F_{q}[x]$ if and only if the homogeneous system of linear equations (12) has a solution $f_{0}, f_{1}, \ldots, f_{l}, g_{0}, g_{1}, \ldots, g_{e}$ in which not all of the $g_{j}$ vanish.

\section{REFERENCES}

1. A. Weil, Basic Number Theory (Springer-Verlag, Heidelberg, 1967; Mir, Moscow, 1972).

2. Z. I. Borevich and I. R. Shafarevich, Number Theory (Nauka, Moscow, 1964) [in Russian]. 